\title{
DISIR: DEEP IMAGE SEGMENTATION WITH INTERACTIVE REFINEMENT
}

\author{
G. Lenczner ${ }^{1,2, *}$, B. Le Saux ${ }^{1}$, N. Luminari ${ }^{2}$, A. Chan-Hon-Tong ${ }^{1}$, G. Le Besnerais ${ }^{1}$ \\ ${ }^{1}$ ONERA / DTIS, Université Paris-Saclay, F-91123 Palaiseau, France \\ ${ }^{2}$ Delair, FR-31400 Toulouse, France
}

KEY WORDS: Semantic Segmentation, Deep Neural Networks, Interactive, Aerial Images, Optical imagery, Human-in-the-loop

\begin{abstract}
:
This paper presents an interactive approach for multi-class segmentation of aerial images. Precisely, it is based on a deep neural network which exploits both RGB images and annotations. Starting from an initial output based on the image only, our network then interactively refines this segmentation map using a concatenation of the image and user annotations. Importantly, user annotations modify the inputs of the network - not its weights - enabling a fast and smooth process. Through experiments on two public aerial datasets, we show that user annotations are extremely rewarding: each click corrects roughly 5000 pixels. We analyze the impact of different aspects of our framework such as the representation of the annotations, the volume of training data or the network architecture. Code is available at this address ${ }^{\dagger}$.
\end{abstract}

\section{INTRODUCTION}

Computer vision has seen tremendous progress in the last few years thanks to the emergence of powerful deep learning algorithms. This results in almost mature algorithms which are now used in industry. However, the devil is in the details and it is often not possible to reach the precision expected by industrial end-users. To fully automate computer vision tasks, a human supervision is still often necessary to assert the quality of the results. We focus in this paper on semantic segmentation of aerial images. This task consists in image classification at the pixel level and is useful in remote sensing and Earth observation to monitor man-made architectures or natural phenomena. Using deep learning tools, it has been first addressed with fully convolutional networks in (Long et al., 2015) and is now efficiently tackled with powerful convolutional neural networks (CNNs) such as Deeplabv3+ (Chen et al., 2018). Under appropriate conditions (e.g. when a large enough training dataset is available), one might say that semantic segmentation is nearly achieved. Indeed, these segmentation algorithms lack only a few percents of precision to reach perfect scores on public benchmarks. However, these few percents can visually make a big difference and therefore not be tolerable in practice. Besides, it often gets worse in real-life datasets due to a variety of factors (complex data, lack of wellannotated ground-truth, various usage domains, ...). This paper proposes a fast procedure to iteratively refine the segmentation maps with a human in the loop. It consists in a neural network pre-trained with simulated human annotations and which does not require any retraining during the interactive process.

In order to concretely motivate our approach, let us consider two famous aerial image datasets in remote sensing. On the INRIA Aerial Image Labelling Dataset (Maggiori et al., 2017), a building segmentation dataset, the current best networks reach an Intersection over Union (IoU) around 0.8 and a pixel accuracy around $97 \%$ on the test set. On the ISPRS Potsdam multi-class segmentation dataset (Rottensteiner et al., 2012), the state-of-the-art approaches almost reach a pixel accuracy of $92 \%$ on the test set. While these performances are incredibly high, there might still remain some misclassified areas unacceptable for an end-user. Besides, these optimal results are obtained using top notch neural

\footnotetext{
* Corresponding author

${ }^{\dagger}$ https://github.com/delair-ai/DISIR
}

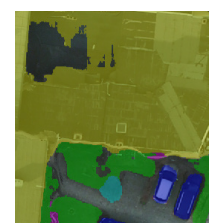

1 - Initial segmentation

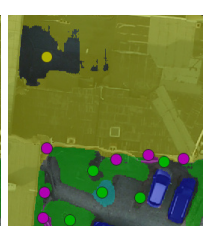

2 - Annotation phase

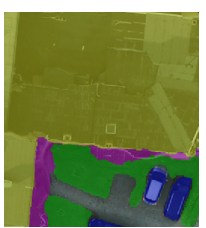

3 - Refined segmentation

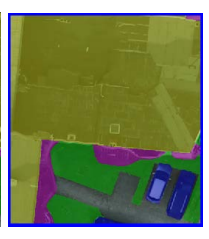

Ground-truth
Figure 1. Example of the proposed interactive semantic segmentation approach on the ISPRS Postdam multi-class dataset (Rottensteiner et al., 2012)

networks which have required many specific refinements (Yue et al., 2019). An off-the-shelf neural network still yields good results but, as the baselines show, a drop of performance between 5 and $10 \%$ can be expected. Moreover, these performances decrease quickly when the networks are faced to the domain shift issues inherent to machine learning. Therefore, the segmentation masks output by these neural networks have to be manually reviewed to meet the expectations of a potential end-user.

Let us also consider a practical application for which current approaches still yield imperfect results. Drones are increasingly used to monitor different environments like crop fields, railroads or quarries. In this context, semantic segmentation can be extremely useful for different tasks such as defects detection, volumes computation or crop monitoring. However, due to the complexity and the high variety of the acquisitions, results are usually not as good as on public datasets while a high precision is necessary for these tasks. Therefore, the operators often have to manually refine the segmentation maps which is a slow process.

To address these issues, we propose to adopt an interactive semantic segmentation approach, as sketched in Figure 1. Indeed, a human in the loop can easily spot the misclassified areas and correct them thanks to a more complex yet intuitive analysis. The difficulty then is to reach optimal classification while keeping the whole process swift and engaging enough.

Our present contribution is as follows.

1. We propose an interactive segmentation framework for aerial images using deep learning. Once the classic train- 
ing phase of a neural network is done, our algorithm does not need any retraining and can therefore quickly refine the segmentation maps.

2. Built upon previous computer vision work mostly oriented toward interactive binary segmentation, our work focuses on interactive multi-class segmentation

3. We study the relevance of this approach under different conditions. Exhaustively, we investigate the impact of annotation encoding, annotation positioning, training set size and architecture choice.

The rest of this paper is organized as follows. We first review the related work in the end of this section, second present our approach in Section 2., then discuss how to evaluate it in Section 3. and finally detail our experiments in Section 4.

\subsection{Review of related work}

Interactive interpretation of remote sensing data has a long history, partially due to the lack of reference data for training in that field. Interactivity has been processed by various techniques to enhance data mining tools with relevance feedback capability : Bayesian modeling of sample distributions was at the core of VisiMine (Aksoy et al., 2004), and Support Vector Machines (SVMs) were used in (Ferecatu and Boujemaa, 2007). More recently, boosting has been the method of choice due to the possibility to train quickly in an incremental manner (dos Santos et al., 2013; Le Saux, 2014). Active learning, or in other words looking for examples which are the more able to lead to a better classification, has also been used for this purpose (Tuia et al., 2009; Bruzzone and Persello, 2009). With respect to these works, our approach apply deep learning for interactive remote sensing.

Interactive segmentation has been tackled in computer vision with a large variety of methods in the last two decades. Older ones are usually graph based methods (Boykov and Jolly, 2001; Rother et al., 2004; Grady, 2006) or based on random forests (Saffari et al., 2009; Santner et al., 2009). More recently, best segmentation performances were obtained with CNN-based architectures. So, they are favored to provide the initial segmentation. Several works have then tried to make them interactive to get finer results. They standardly use points resulting from user clicks as annotations. We now review thoroughly these methods.

Deep interactive object selection (DIOS) (Xu et al., 2016) is the first proposal of an interactive segmentation framework based on neural networks. It aims for binary classification. In a nutshell, the network takes as input two additional channels concatenated with the RGB image. The first one contains annotation points from the foreground while the other one contains background points. These annotation points are encoded into euclidean distance maps. The annotations are automatically sampled during training using the ground-truth maps. We extend this approach to multi-class segmentation of aerial images. Multiple existing works are inspired by DIOS. (Liew et al., 2017) adopt a multiscale strategy which refines the global prediction by combining it with local patch-based classification. (Hu et al., 2019) also follow a multi-scale strategy by designing a two-stream fusion network to process the annotations differently than the image.A particular challenge is to get enough useful annotations. For this purpose, (Mahadevan et al., 2018) use a hard-sample mining strategy at training by selecting annotations among erroneous predictions.Alternatively, (Jang and Kim, 2019) iteratively optimize the annotation maps given as inputs by back-propagating the errors between predictions and annotations. Finally, DEXTR (Maninis et al., 2018) and (Wang et al., 2019b) both ask the user to click points on the borders and corners of the objects. Recently, (Benenson et al., 2019) assess these various strategies in the first large scale study of interactive instance segmentation with human annotators. Their experiments hint that center annotation clicks are the most robust and that distance transform to encode the annotation points can be replaced by binary disks.

Polygon-RNN++ (Acuna et al., 2018) is an interesting alternative to DIOS-like approaches. Using a CNN-RNN architecture, they predict a polygon which can be refined by moving its vertices. Using Graph Convolutional Networks (GCN), Curve-GCN (Ling et al., 2019) extend this work by predicting a spline which better outlines curved objects. Note that these aforementioned approaches aim to binary classification.

Multi-class interactive segmentation has also been approached in various ways. Several older methods (Nieuwenhuis et al., 2014; Nieuwenhuis and Cremers, 2012) address this problem using a bayesian maximum a posteriori (MAP) approach while (Santner et al., 2010) rely on a random forest classifier. Recently, (Andriluka et al., 2018) use a combination of two slightly modified Mask-RCNN (He et al., 2017) to compute multiple fixed segmentation propositions and then let the user choose which of these propositions should form the final segmentation. Finally, (Agustsson et al., 2019) are the first to propose a deep learning approach which lets the user correct the shape of a proposed multi-class segmentation. Their algorithm takes as input a concatenation of the image and the extreme points of each instance in the scene and then corrects the segmentation proposal using scribbles. In contrast, we adopt in our work a class-dependant DIOS-based approach to refine an initial segmentation map.

Automatic evaluation of an interactive system requires some way of mimicking the human annotation. (Xu et al., 2016) try to mimick a user who would correct the largest mislabelled regions by iteratively placing the annotations far from the boundaries of the mislabelled prediction. In details, they use as annotation the point which maximises the distance to the boundary of the false prediction in order to simulate an image analyst. Then, they do a new prediction using this generated annotation and repeat this process for 20 iterations. Our automatic clicking strategy is inspired from this idea and adapted to our multi-label segmentation problem. (Benenson et al., 2019) add some noise in the simulated clicks to better match the human behavior. They also manually experiment their approach with a large pool of human annotators. In 3., we investigate different strategies to simulate human analysts and evaluate automatically an interactive system.

\section{PROPOSED ALGORITHM}

We now describe in details the proposed approach for interactive multi-class segmentation of aerial images. In particular, our goal is to train a neural network with two purposes:

1. producing an initial high quality segmentation map of the scene without any external help;

2. using annotations provided by an operator to quickly enhance its initial prediction.

To achieve this, we propose a neural network which keeps its original structure but takes as input a concatenation of the classic inputs (e.g. RGB) and of the annotations ( $N$ channels, one per class). These annotations are clicked points. Note that only the inputs of the network are modified and not its weights: this makes 


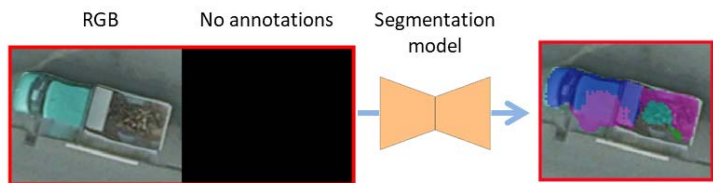

Initialization phase

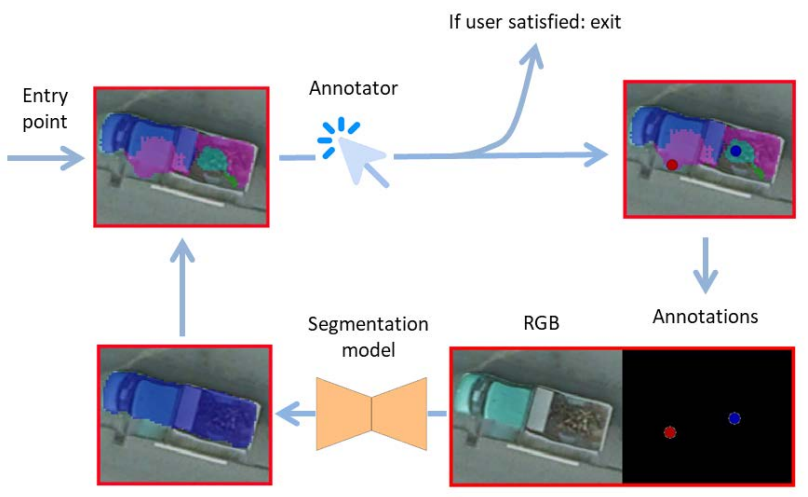

Interactive loop

Figure 2. High level overview of the proposed approach: information provided by the user modifies the input of the network - not the network itself - allowing an effective interaction

the swiftness of the approach. Figure 2 presents a high-level overview of our approach.

We first define our training strategy and then present our study on the annotations themselves.

\subsection{Training strategy}

In the following, we assume that we have a segmentation reference composed of $N$ classes. Ground-truth maps are the core of our training strategy. On one hand, they are classically used to compute and back-propagate the loss. On the other hand, they are also randomly sparsified to sample annotations. In other words, only a few pixels from the ground-truth are kept to be used as annotations. According to their class, these annotations are encoded in the $N$ annotation channels given as input to the algorithm. To train under various annotation layouts, the number of sampled annotations is random in each training example. Since the network has to be able to create an accurate segmentation map without them, the possibility of a lack of annotations is also sampled. Concretely, this situation means that the annotation channels are filled with zeros.

If the annotations are sampled independently of their class, the following problem may occur. During the evaluation phase, annotations on sub-represented classes can be ignored by the network because it has barely seen any annotation points of these classes during training. Therefore, it has not learned how to use them to enhance its predictions. To overcome this issue, we use a frequency balancing strategy to sample the annotations based on the classes distributions. It allows the network to equally see annotations from each class during training and, therefore, to be efficiently guided once the training is done.

\subsection{Annotation representation}

We investigate two aspects of the annotation representation: how to position clicks in order to sample the most useful information, and how to encode clicks to get the best benefit.

Click positioning. Fixing a wrong segmentation implies to provide the system with additional information about the right division. New samples provided by clicks may represent either the inside of an instance or its border.

The first case seems to be the most intuitive. Clicked pixels are inside instances and the annotation points represent the class associated to these instances. Contrary to (Xu et al., 2016), we do
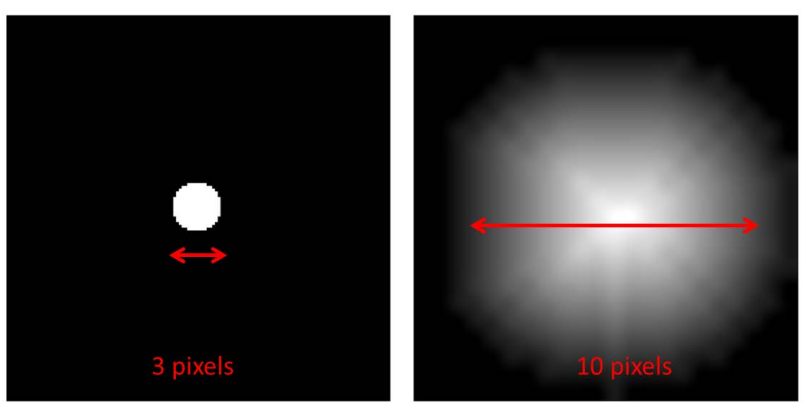

Figure 3. Binary (left) and distance transform (right) click.

not sample them at a minimal distance from the boundaries since we assume that an annotator might click near an edge to fine-tune the prediction. For the second case where the annotations represent the borders of the instances, the channel associated to a click corresponds to a class randomly chosen among the ones adjacent to the clicked border.

Aiming to ease the burden of the end users, we also explored softer constraints on the annotations. Indeed, instead of using $N$ annotation channels, we summarized them into a single annotation channel. For the border strategy, this single channel only indicates the presence of a border. For the inside point strategy, it only indicates where the network has initially made a mistake. To implement this latter strategy, we had to slightly modify the training process. The network performs a first inference to create a segmentation map used to find mislabelled regions. Annotations are then sampled in these areas and a second inference is performed. Only this second inference is used to back-propagate the gradients. However, as shown in Section 4.4, none of these simplified annotations seems promising to efficiently guide the segmentation task.

Click encoding. User clicks can be encoded in various ways, and such may provide the system with more or less spatial information, as shown in Figure 3. In particular, we consider:

- Small binary area around the annotation points

- Euclidean distance transform maps around these points

As shown in Section 4., the inside point strategy with distance transform encoding seems to be our most successful combination. 


\section{DISCUSSION ON THE EVALUATION STRATEGY}

To evaluate interactive approaches, two procedures are possible. Either the ground-truth is available and can be used to sample annotations, or it is not and a human operator has to make the annotations. Therefore, we have evaluated our approach both manually and automatically. We use the IoU averaged over all classes as our evaluation metric.

Automatic evaluation For each image, the neural network makes a first inference without annotations. A click is then automatically generated and the network makes a new inference. This process is repeated iteratively for a fixed number of clicks.

We have compared two click sampling strategies, both using a comparison between the prediction and the ground-truth:

1. A click is automatically sampled in one of the biggest mislabelled areas. Some randomness is added in the choice of the area and in the localization of the click inside the area to better simulate a human behavior. This process is classindependent.

2. The process is similar to 1 . but the generated click has the supplementary constraint to be on a pixel belonging to a specified class. This allows to also correct pixels belonging to sub-represented classes. This process is class-dependent.

As we will see in 4.2 , the class-dependent process is better than the first one to evaluate the influence of the clicks on subrepresented classes but has two drawbacks: overall smaller erroneous areas are corrected which leads to smaller corrections and there is less room for randomness if the chosen class to annotate is predetermined.

Manual evaluation This process is similar to the automatic one but the clicks are now made by a human operator. This operator also aims to correct the biggest erroneous areas but the localization of the clicks is now inherently subjective.

To do this manual evaluation, we have built a QGIS (QGIS Development Team, 2009) plugin. User interaction is then handled by the QGIS interface while the heavy computations, e.g. the semantic segmentation, are performed in a separate server that can be local or remote. Once the server is launched, the data transfer is transparent to the user.

\section{EXPERIMENTS}

In this section, we aim to show that our method works and how to best evaluate it among the two evaluation strategies aforementioned in Section 3.. Besides, we study the influence of the different parameters described in Section 2.2. Furthermore, we conduct two experiments to better apprehend the possibilities and the limits of our approach:

- We have first compared different backbone architectures to evaluate if it has a significant impact on the performances. More importantly, since these different architectures produce different initial segmentation maps, this comparison also allows us to study if the initial quality of the segmentation maps influences the benefits brought by the annotations.
- The second one is motivated by the fact that it often happens in practice to only have access to a very limited amount of annotated data. Similar to (Castillo-Navarro et al., 2019) where the authors study the influence of the training set size on the network performance, we study the influence of this parameter on the neural network refinement abilities. To this end, we have trained the networks on subsets of the initial training sets.

This section is thus organized as follows. We first present our experimental setup. Second, we show that our method works and how to best evaluate it. Then, we analyse the outcomes of our different experiments with automatic evaluation. Finally, we draw conclusions from the manual evaluations.

\subsection{Experimental setup}

Datasets. We have tested our approach on the two standard remote sensing datasets mentionned in the Introduction. We split the initial training sets into a training and a validation sets with a $80 \%-20 \%$ ratio and use the validation sets for our experiments. The INRIA Aerial Image Labelling dataset (Maggiori et al., 2017) is composed of two classes (buildings and not buildings) and covers more than $800 \mathrm{~km}^{2}$ with a spatial resolution of $0.3 \mathrm{~m}$. The size of each image is $5000 \times 5000$ pixels. The training set is composed of 144 images and the validation set of 36 images. The ISPRS Potsdam dataset (Rottensteiner et al., 2012) is composed of 6 classes (impervious surface, buildings, low vegetation, tree, car and clutter). The class car is sub-represented compared to the other classes. This dataset covers around $3 \mathrm{~km}^{2}$ with a spatial resolution of $0.05 \mathrm{~m}$. The size of each image is $6000 \times 6000$ pixels. The training set is composed of 19 images and the validation set of 5 images.

Neural Network. Except in the backbone comparison, we use a LinkNet (Chaurasia and Culurciello, 2017) architecture. It is a classic encoder/decoder architecture relying on a ResNet encoder (He et al., 2016). The networks are trained using stochastic gradient descent (SGD) and cross-entropy loss for 50 epochs with a batch of size 8, seeing during each epoch 10000 samples randomly chosen and cropped (size $512 \times 512$ ). The initial learning rate is fixed at 0.05 and is divided by 10 after 15,30 and 45 epochs. Only basic data augmentation is performed: horizontal and vertical flips. The implementation is done using Pytorch.

Annotations. During our different evaluations, we sample 120 clicks for each image and measure the IoU gain for each class. Except when specified otherwise, we encode clicks using distance transform and assume that they represent the inside of the corrected instances. The annotations for the Potsdam dataset are sampled during training based on their class distribution, except in the frequency balancing influence study.

\subsection{Approach assessment and automatic evaluation strategy}

Here we compare the two proposed automatic evaluation strategies. Let us recall that, for the first strategy, the clicks are sampled iteratively in the biggest erroneous areas independently of the class while they are sampled equally in each class in the second one. As we can see on Figure 4, the class independent evaluation strategy allows to reach a higher overall IoU but to the detriment of the sub-represented car class in the Potsdam dataset. This is due to the fact that the biggest erroneously predicted areas inherently belong to larger instances than cars such as buildings. Therefore, even though the overall metric gain is not as good as with the class-independent evaluation process, we choose the class-dependent one for our further evaluations on Potsdam. 

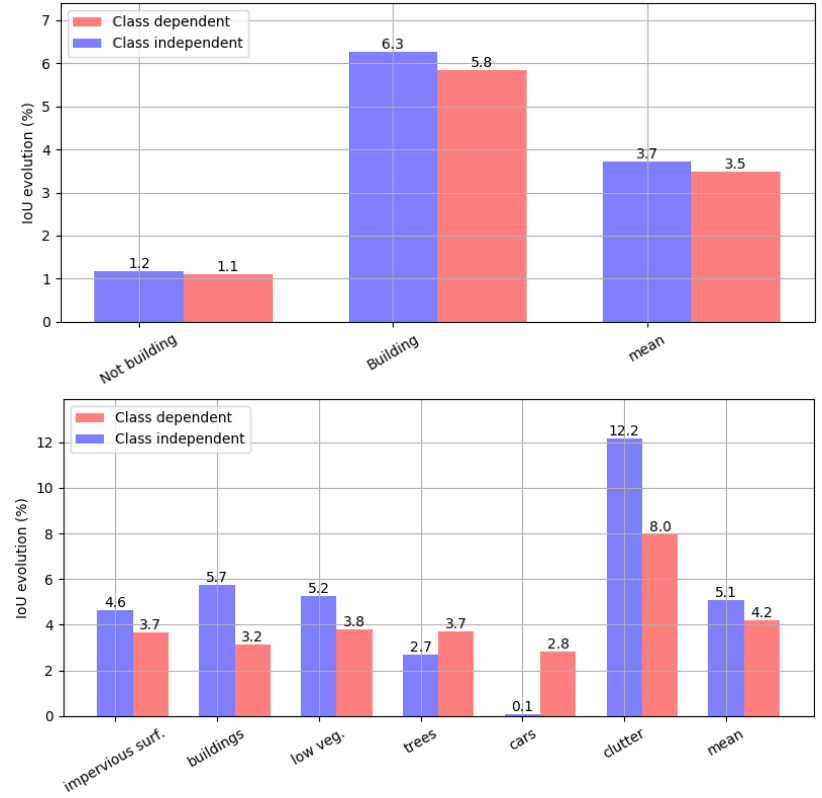

Figure 4. Comparison of the two different automatic evaluation processes on the INRIA (top) and Potsdam (bottom) datasets.

\begin{tabular}{c|c} 
Dataset & Corrected pixels \\
\hline INRIA & 3143 \\
Potsdam & 7219
\end{tabular}

Table 1. Average corrected pixels per click

However, the INRIA dataset does not contain a low-represented class so we choose the class-independent evaluation process to evaluate our experiments on this dataset.

Whatever the evaluation strategy, the results displayed in Figure 4 validate the efficiency of our approach on both datasets. Indeed, all segmentation performances are improved for all classes: on average the mean IoU is increased by $3.7 \%$ on the INRIA Building dataset, and by $4.2 \%$ on the muti-class ISPRS Potsdam dataset. Besides, as we can see on Table 1, each click allows to correct around 5000 pixels in average.

\subsection{Influence of the frequency balancing}

Figure 5 compares the improvements between training with frequency balancing to sample the annotations and without. Here, we use binary-encoded clicks in order to control the number of samples per class. As we can see, without frequency balancing, the network does not learn to use car annotations to refine its predictions. Indeed, the low-representation of this class implies that only few annotations from it were seen during training. A frequency balancing strategy efficiently tackles this issue. Overall, mean IoU is increased by almost $1 \%$ and 5 classes out of 6 are improved. However, frequency balancing is not necessary for the INRIA dataset which does not contain any low-represented class.

\subsection{Influence of the annotation strategy}

We compare here the different annotation strategies: inside clicks or border clicks, binary encoding or distance transform, and single or multiple channels. As we can see on Figure 6, the distance transform clearly increases the benefits of the annotations compared to the binary encoding. While (Benenson et al., 2019) conclude that the binary encoding leads to better performances, our opposite conclusion might be inherent to the large size and

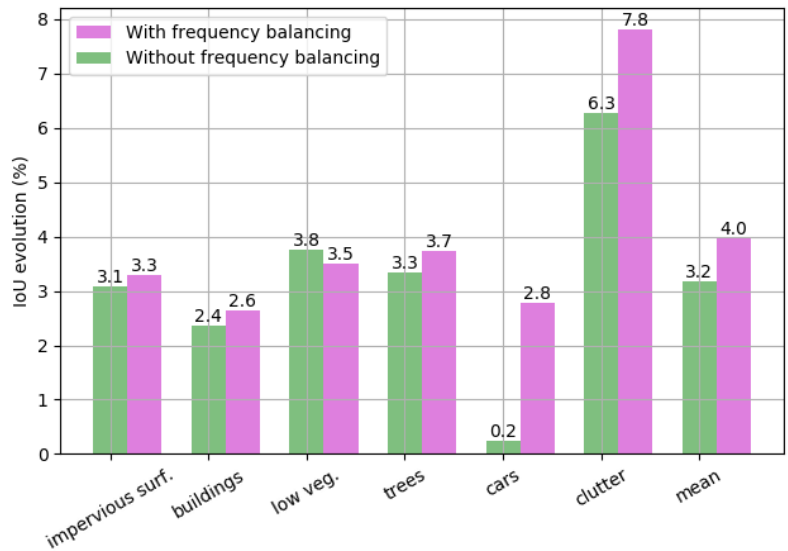

Figure 5. Study of the impact of the frequency balancing during training on the Potsdam validation set.
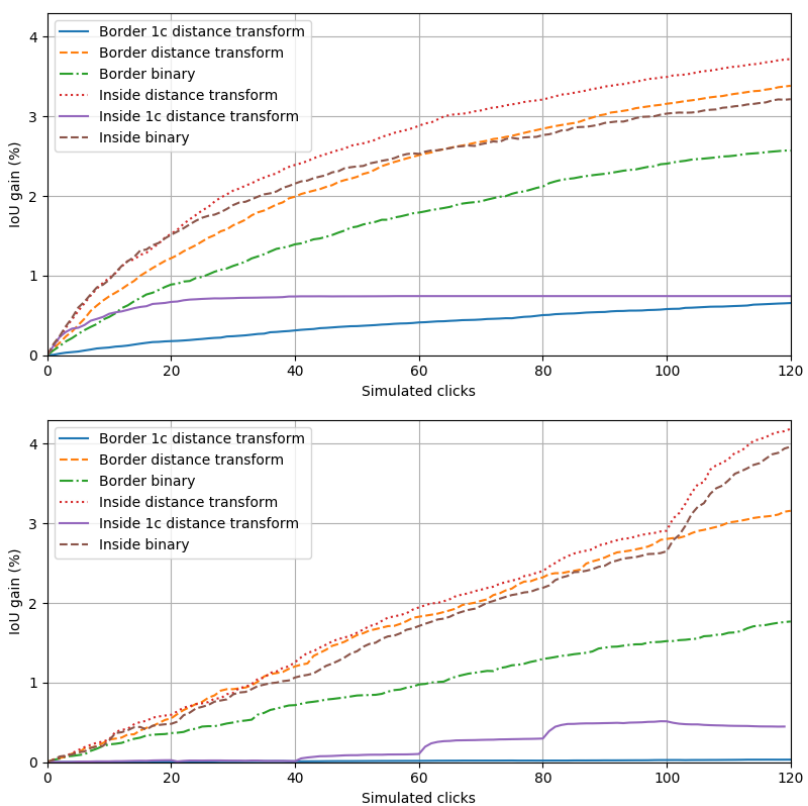

Figure 6. Comparison of the different annotation strategies on the INRIA (top) and Potsdam (bottom) datasets. $1 c$ means single annotation channel.

scale of aerial images which dilute the annotations localized over very small areas.

Both the contours and the inside points are efficiently used by the network to enhance its predictions but it is still noticeably better with the inside points. We can also notice that the last 20 added points considerably boost the performances of the inside point strategies for the Potsdam dataset: this is due to the fact that these points belong to the class clutter, an under-represented class. Therefore, they have the strongest impact in term of IoU. Finally, the two degraded strategies which rely on single annotation channel bring little or no improvement even though it is slightly better for the INRIA dataset since it contains only two classes.

\subsection{Influence of the network backbone}

We compare LinkNet to SegNet (Badrinarayanan et al., 2017), UNet (Ronneberger et al., 2015) and DeepLabv3 (Chen et al., 


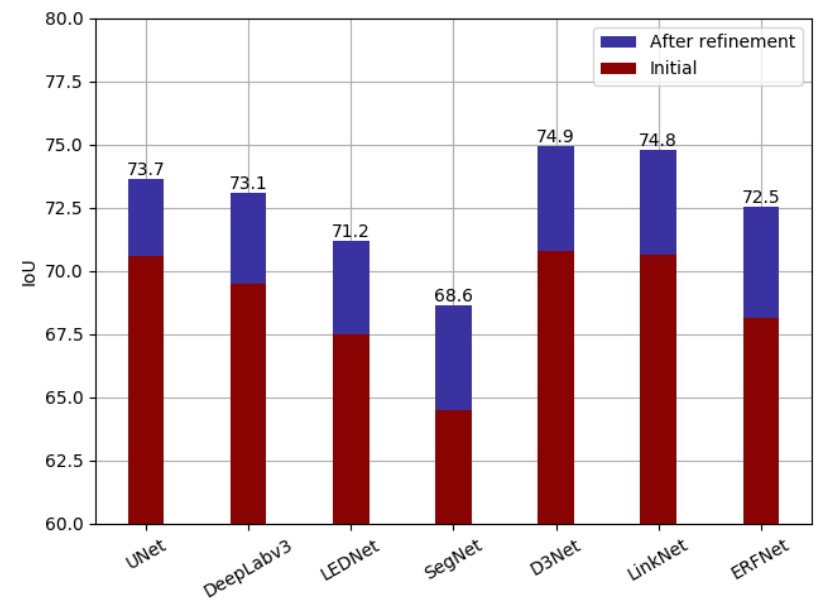

Figure 7. Study of the impact of the architecture choice on the Potsdam validation set sorted per IoU gain.

2017) which are standard segmentation networks with increasing complexity and also to the following lighter architectures: LEDNet (Wang et al., 2019a), ERFNet (Romera et al., 2017) and D3Net (Carvalho et al., 2018). Figure 7 shows the results obtained with the different architectures under the same training and evaluating conditions. As expected since this framework is agnostic to the network architecture, the gains are in the same order of magnitude. Indeed, the initial IoU mean is $68.8 \%$ with a standard deviation of 2.13 while the IoU gain mean is $3.9 \%$ with a standard deviation of 0.4 . Figure 7 also shows that the accuracy gain of the interactive correction seems to be uncorrelated to the accuracy of the initial segmentation map. For instance, the worse initial architecture here - SegNet - is the average one in regards to the IoU gain.

\subsection{Influence of the volume of training data}
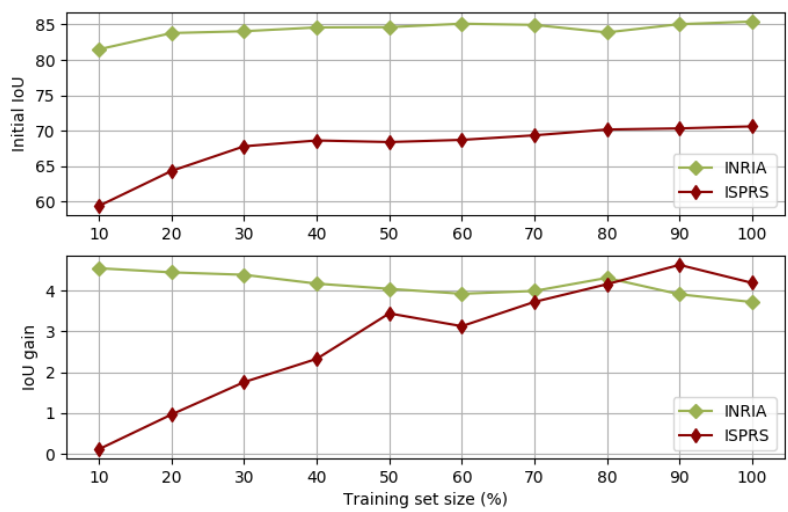

Figure 8. Influence of the training set size on the initial IoU (top) and on the IoU gain (bottom)

Figure 8 shows the influence of the training set size on our approach. The different behavior on the two datasets can be explained by their initial size difference.

On the INRIA dataset, since the initial training size is high, even $10 \%$ of the training data seems to be enough to provide a network with a decent initial accuracy and a good ability to use the annotations. Besides, if more data implies a better initial accuracy, it does not improve the performances of the interactive correction.
This shows that the network has not learned to make a better use of the annotations with supplementary data.

On the Potsdam dataset, even though the initial training size is lower than in the INRIA dataset, the network is still initially quite accurate with little training data. Indeed, according to the results of (Castillo-Navarro et al., 2019), since there are pictures from only one city, few training images are enough to learn the general semantic of the dataset even if the full training set provides better performances. However, the accuracy gain is really low with little training data. For example, the IoU gain is less than $1 \%$ with $20 \%$ of the initial volume of data while it is slightly over $4 \%$ with the full training set. We believe that this lack of performance in low-data regime is due to over-fitting. Indeed, since there are only a few training images in this scenario, there are also less possible annotations and they might not fully reflect the reality of the test set. Besides, if the network over-fits on these few images, it might also consider the annotations as unnecessary for the segmentation.

Therefore, as shown by the study on the Potsdam dataset, a certain amount of data seems necessary to optimally use the annotations. However, as shown by the study on the INRIA dataset, the network ability to use the annotations reaches a plateau once there is enough available training data.

\subsection{Manual analysis}

In this experiment, the images from the Potsdam validation set have been manually refined by a human annotator. If the number of clicks exceeds 120 , we threshold it at 120 in order to make a fair comparison with the the automatic process.

Local insights. On one hand, as shown on Figure 9, the refinements can be very intuitive and effective on areas semantically similar to the ones seen during training. On the other hand, if the semantic is new compared to what is in the training set, the neural networks have trouble to use the annotations efficiently.

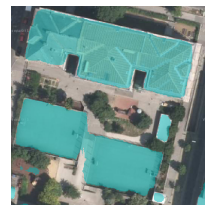

1 - Initial segmentation

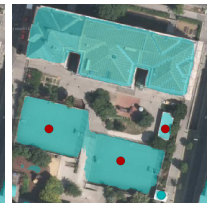

2 - Annotation phase

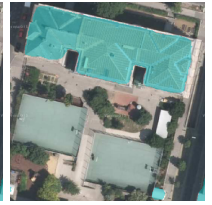

3 - Refined segmentation

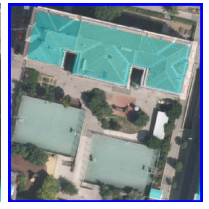

Ground-truth
Figure 9. Annotations lead to an easy false positive buildings removal on the segmentation map

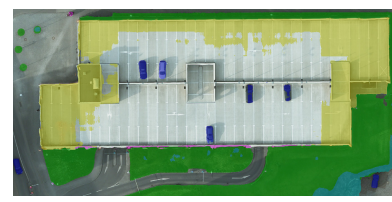

1 - Initial segmentation

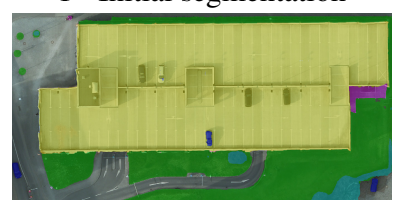

3 - Refined segmentation

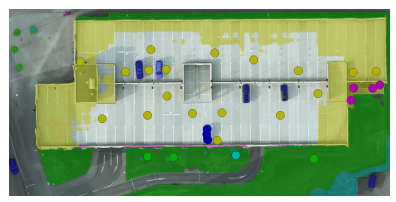

2 - Annotation phase

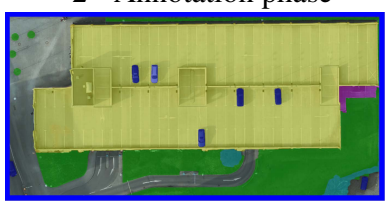

Ground-truth
Figure 10. Difficult segmentation of an outside parking since the network has not learned the semantic cars on building. Only the car at the bottom is annotated and recognized. 
For example, in the Potsdam dataset, there is only one outside car park considered as building which means only one place with the semantic "car" surrounded by "building" in the dataset. We kept the associated image in the validation set to study the impact of the annotations in this scenario. Figure 10 shows the outcome of our approach on this car park. Since it also looks like a road, it is initially difficult for the network to segment it correctly. Nonetheless, it succeeds to recognize the cars parked there. Then, with building annotations, the network successfully recognizes a building. However, it also considers that the vehicles parked there are now part of the building since it has never seen the class "car" surrounded by the class "building" during training. As we can see on Figure 10, with supplementary car annotations, the network can still recognize the correct semantic of the scene. However, the process in this case is not very smooth and intuitive since the cars which were primarily well recognized need to be annotated nonetheless. This example shows that our framework does not perform optimally when it is faced to areas with a different semantic compared to the ones present in the training set.
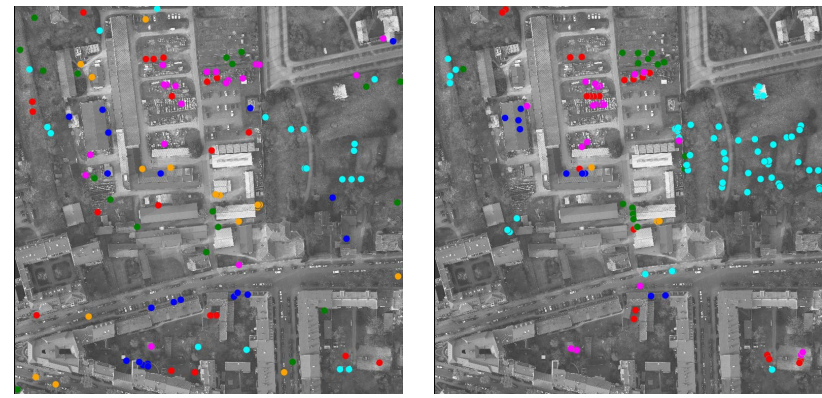

Figure 11. Click distribution on an image from Potsdam in automatic (left) and manual (right) evaluations. The colors represent the different classes.

General insights. Regarding the click distribution, as shown in Figure 11, a human operator tends to focus clicking on specific areas while the automatic evaluation rather spreads the annotations all across the image. However, as shown in Figure 12, these grouped clicks seem to efficiently increase the metric. Indeed, with the manual evaluation, 4 classes out of 6 are more improved and the mean IoU gain is overall better. This shows the efficiency of our approach with a real user in the loop. Finally, Figure 13 shows qualitative results before and after human interaction.

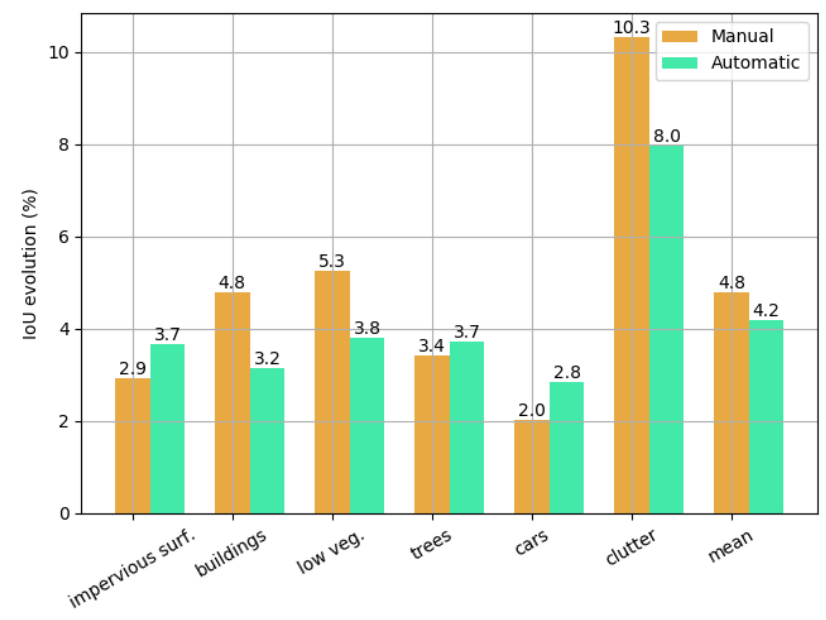

Figure 12. Comparison of the IoU evolution between an automatic and a manual evaluation on the Potsdam dataset.

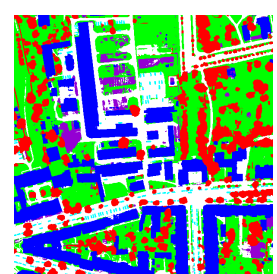

Before HI $(87.5 \%)$

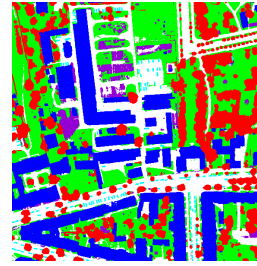

After HI (90.4\%)

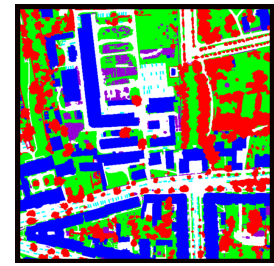

Ground-truth
Figure 13. Full predictions and their accuracy before/after human interaction (HI) on an image from Potsdam

\section{CONCLUSION}

We have proposed in this article an interactive multi-class segmentation framework for aerial images. Starting from a neural network designed for semantic segmentation purpose, it consists in training this network to exploit user annotation inputs. At testing time, user annotations are input in the neural network without changing the parameters of the model, hence the interactive semantic segmentation process is swift and efficient. Through experiments on two public aerial datasets, we have shown that interactive refinement is efficient for all classes. It improves classification results by $4 \%$ on average for 120 clicks and mainly, produces segmentation maps which are visually more rewarding. We have shown that our interactive process is efficient whatever the network backbone is. We have also investigated different representations of the annotations and have concluded that clicks positioned inside instances and encoded using distance transform carry the most meaningful information. In the future, we will further investigate class-dependent annotation encoding.

\section{REFERENCES}

Acuna, D., Ling, H., Kar, A. and Fidler, S., 2018. Efficient interactive annotation of segmentation datasets with PolygonRNN++. In: Conference on Computer Vision and Pattern Recognition (CVPR), IEEE, pp. 859-868.

Agustsson, E., Uijlings, J. R. and Ferrari, V., 2019. Interactive full image segmentation by considering all regions jointly. In: Conference on Computer Vision and Pattern Recognition (CVPR), IEEE, pp. 11622-11631.

Aksoy, S., Koperski, K., Tusk, C. and Marchisio, G., 2004. Interactive training of advanced classifiers for mining remote sensing image archives. In: Knowledge Discovery in Databases (KDD), ACM, pp. 773-782.

Andriluka, M., Uijlings, J. R. and Ferrari, V., 2018. Fluid annotation: a human-machine collaboration interface for full image annotation. In: MultiMedia (MM), ACM, pp. 1957-1966.

Badrinarayanan, V., Kendall, A. and Cipolla, R., 2017. SegNet: A deep convolutional encoder-decoder architecture for image segmentation. In: Trans. on Pattern Analysis and Machine Intelligence (TPAMI), Vol. 39, no. 12, IEEE, pp. 2481-2495.

Benenson, R., Popov, S. and Ferrari, V., 2019. Large-scale interactive object segmentation with human annotators. In: Conference on Computer Vision and Pattern Recognition (CVPR), IEEE, pp. 11700-11709.

Boykov, Y. Y. and Jolly, M.-P., 2001. Interactive graph cuts for optimal boundary \& region segmentation of objects in nd images. In: International Conference on Computer Vision (ICCV), IEEE, pp. 105-112.

Bruzzone, L. and Persello, C., 2009. Active learning for classification of remote sensing images. In: International Geoscience and Remote Sensing Symposium (IGARSS), IEEE, pp. 689-693. 
Carvalho, M., Le Saux, B., Trouvé-Peloux, P., Almansa, A. and Champagnat, F., 2018. On regression losses for deep depth estimation. In: International Conference on Image Processing (ICIP), IEEE, pp. 2915-2919.

Castillo-Navarro, J., Audebert, N., Boulch, A., Le Saux, B. and Lefèvre, S., 2019. What data are needed for semantic segmentation in earth observation? In: Joint Urban Remote Sensing Event (JURSE), IEEE.

Chaurasia, A. and Culurciello, E., 2017. LinkNet: Exploiting encoder representations for efficient semantic segmentation. In: Visual Communications and Image Processing (VCIP), IEEE.

Chen, L.-C., Papandreou, G., Schroff, F. and Adam, H., 2017. Rethinking atrous convolution for semantic image segmentation. In: arXiv preprint arXiv:1706.05587.

Chen, L.-C., Zhu, Y., Papandreou, G., Schroff, F. and Adam, H., 2018. Encoder-decoder with atrous separable convolution for semantic image segmentation. In: European Conference on Computer Vision (ECCV), Springer, pp. 801-818.

dos Santos, J. A., Gosselin, P., Philipp-Foliguet, S., Torres, R. d. S. and Falcão, A. X., 2013. Interactive multiscale classification of high-resolution remote sensing images. In: Journal of Selected Topics in Applied Earth Observations and Remote Sensing (JSTARS), Vol. 6, no. 4, IEEE, pp. 2020-2034.

Ferecatu, M. and Boujemaa, N., 2007. Interactive remote-sensing image retrieval using active relevance feedback. In: Trans. on Geoscience and Remote Sensing (TGRS), Vol. 45, no. 4, IEEE, pp. 818-826.

Grady, L., 2006. Random walks for image segmentation. In: Trans. on Pattern Analysis \& Machine Intelligence (TPAMI), Vol. 28, no. 11, IEEE, pp. 1768-1783.

He, K., Gkioxari, G., Dollar, P. and Girshick, R., 2017. Mask RCNN. In: International Conference on Computer Vision (ICCV), IEEE, pp. 2961-2969.

He, K., Zhang, X., Ren, S. and Sun, J., 2016. Deep residual learning for image recognition. In: Conference on Computer Vision and Pattern Recognition (CVPR), IEEE, pp. 770-778.

Hu, Y., Soltoggio, A., Lock, R. and Carter, S., 2019. A fully convolutional two-stream fusion network for interactive image segmentation. In: Neural Networks, Vol. 109, Elsevier, pp. 31-42.

Jang, W.-D. and Kim, C.-S., 2019. Interactive image segmentation via backpropagating refinement scheme. In: Conference on Computer Vision and Pattern Recognition (CVPR), IEEE, pp. 5297-5306.

Le Saux, B., 2014. Interactive design of object classifiers in remote sensing. In: International Conference on Pattern Recognition (ICPR), IEEE, pp. 2572-2577.

Liew, J., Wei, Y., Xiong, W., Ong, S.-H. and Feng, J., 2017. Regional interactive image segmentation networks. In: International Conference on Computer Vision (ICCV), IEEE, pp. 2746-2754.

Ling, H., Gao, J., Kar, A., Chen, W. and Fidler, S., 2019. Fast interactive object annotation with Curve-GCN. In: Conference on Computer Vision and Pattern Recognition (CVPR), IEEE pp. 5257-5266.

Long, J., Shelhamer, E. and Darrell, T., 2015. Fully convolutional networks for semantic segmentation. In: Conference on Computer Vision and Pattern Recognition (CVPR), IEEE, pp. 34313440 .

Maggiori, E., Tarabalka, Y., Charpiat, G. and Alliez, P., 2017. Can semantic labeling methods generalize to any city? the inria aerial image labeling benchmark. In: International Geoscience and Remote Sensing Symposium (IGARSS), IEEE, pp. 32263229.
Mahadevan, S., Voigtlaender, P. and Leibe, B., 2018. Iteratively trained interactive segmentation. In: arXiv preprint arXiv: 1805.04398.

Maninis, K.-K., Caelles, S., Pont-Tuset, J. and Van Gool, L., 2018. Deep Extreme Cut: from extreme points to object segmentation. In: Conference on Computer Vision and Pattern Recognition (CVPR), IEEE, pp. 616-625.

Nieuwenhuis, C. and Cremers, D., 2012. Spatially varying color distributions for interactive multilabel segmentation. In: Trans. on Pattern Analysis and Machine Intelligence (TPAMI), Vol. 35, no. 5, IEEE, pp. 1234-1247.

Nieuwenhuis, C., Hawe, S., Kleinsteuber, M. and Cremers, D., 2014. Co-sparse textural similarity for interactive segmentation. In: European Conference on Computer Vision (ECCV), Springer, pp. 285-301.

QGIS Development Team, 2009. QGIS Geographic Information System. Open Source Geospatial Foundation.

Romera, E., Alvarez, J. M., Bergasa, L. M. and Arroyo, R., 2017. ERFNet: Efficient residual factorized convnet for real-time semantic segmentation. In: Trans. on Intelligent Transportation Systems (ITS), Vol. 19, no. 1, IEEE, pp. 263-272.

Ronneberger, O., Fischer, P. and Brox, T., 2015. U-Net: Convolutional networks for biomedical image segmentation. In: International Conference on Medical Image Computing and ComputerAssisted Intervention (MICCAI), Springer, pp. 234-241.

Rother, C., Kolmogorov, V. and Blake, A., 2004. GrabCut: Interactive foreground extraction using iterated graph cuts. In: Trans. On Graphics (TOG), Vol. 23, no. 3, ACM, pp. 309-314.

Rottensteiner, F., Sohn, G., Jung, J., Gerke, M., Baillard, C., Benitez, S. and Breitkopf, U., 2012. The ISPRS benchmark on urban object classification and 3D building reconstruction. In: ISPRS Annals of the Photogrammetry, Remote Sensing and Spatial Information Sciences (ISPRS Annals), Vol. 1, no. 1, Göttingen: Copernicus GmbH, pp. 293-298.

Saffari, A., Leistner, C., Santner, J., Godec, M. and Bischof, H., 2009. On-line random forests. In: International Conference on Computer Vision (ICCV), IEEE, pp. 1393-1400.

Santner, J., Pock, T. and Bischof, H., 2010. Interactive multilabel segmentation. In: Asian Conference on Computer Vision (ACCV), Springer, pp. 397-410.

Santner, J., Unger, M., Pock, T., Leistner, C., Saffari, A. and Bischof, H., 2009. Interactive texture segmentation using random forests and total variation. In: British Machine Vision Conference (BMVC), BMVA, pp. 1-12.

Tuia, D., Ratle, F., Pacifici, F., Kanevski, M. and Emery, W., 2009. Active learning methods for remote sensing image classification. In: Trans. on Geoscience and Remote Sensing (TGRS), Vol. 47, no. 7, IEEE, pp. 2218-2232.

Wang, Y., Zhou, Q., Liu, J., Xiong, J., Gao, G., Wu, X. and Latecki, L. J., 2019a. LEDNet: A lightweight encoder-decoder network for real-time semantic segmentation. In: arXiv preprint arXiv:1905.02423.

Wang, Z., Acuna, D., Ling, H., Kar, A. and Fidler, S., 2019b. Object instance annotation with deep extreme level set evolution. In: Conference on Computer Vision and Pattern Recognition (CVPR), IEEE, pp. 7500-7508.

Xu, N., Price, B., Cohen, S., Yang, J. and Huang, T. S., 2016. Deep interactive object selection. In: Conference on Computer Vision and Pattern Recognition (CVPR), IEEE, pp. 373-381.

Yue, K., Yang, L., Li, R., Hu, W., Zhang, F. and Li, W., 2019. TreeUNet: Adaptive tree convolutional neural networks for subdecimeter aerial image segmentation. In: ISPRS Journal of Photogrammetry and Remote Sensing (IJPRS), Vol. 156, Elsevier, pp. 1-13. 